\title{
Symptoms in 298 infants under 6 months old, seen at home
}

\author{
A J Thornton, C J Morley, P H Hewson, T J Cole, M A Fowler, J M Tunnacliffe
}

\begin{abstract}
Symptoms were assessed in 298 infants under 6 months old seen at home. Their mothers were asked if they were concerned about their babies and then questioned about the presence, duration, and severity of 28 predefined symptoms. Forty seven mothers expressed concern about their babies $(16 \%)$, whereas on direct questioning $241(81 \%)$ reported symptoms present within the last 24 hours. One hundred and twenty three $(41 \%)$ reported three or more. Many of the symptoms occurred in more than 15 babies $(5 \%)$. The commonest were cold peripheries $(117,39 \%)$, noisy breathing $(88,30 \%)$, and rash $(68,23 \%)$. These had often been present for many days. Only $257 / 819$ reported symptoms (31\%) were of recent onset ( $<4$ days) with $168(21 \%)$ graded by the mother as moderate or severe. A few highly specific symptoms that were associated with serious conditions were rare Symptoms cannot be interpreted without detailed questioning taking their severity and duration into account, and most occur too often to be used as indicators for referral.
\end{abstract}

After the neonatal period, there is a higher incidence of illness and death in the first six months than in the rest of childhood. It has been suggested that earlier recognition of important symptoms in young babies, and early treatment, might reduce the severity of illness and prevent some deaths. ${ }^{12}$ The multicentre study of postneonatal mortality investigated 297 infants with signs of illness in the days immediately before death and concluded that $24 \%$ of the families had acted 'inappropriately' in the management of their child's illness by not recognising that the child was seriously ill or by failing to seek help. ${ }^{3}$ The general practitioner was contacted in only 131 cases, but in $64 \%$ was deemed to have managed the case 'inadequately'. There was a 'delay in diagnosis' or 'inappropriate treatment' in $26 \%$ of the babies admitted to hospital.

To facilitate the recognition of serious illness Stanton et al highlighted 12 'major' symptoms which needed medical attention the same day. The paper was criticised because the symptoms were considered to be too common to be useful predictors. ${ }^{5-8}$ Valman subsequently suggested four 'undoubtedly sinister' symptoms that warranted an opinion from a paediatrician in an infant under 6 months old. ${ }^{9}$ A further study in general practice, however, suggested that at least two of these symptoms (poor feeding and difficulty in breathing) were 'too common in infants at home to be regarded as truly sinis- ter' ${ }^{10}$ This controversy shows the dearth of accurate data about the incidence and severity of symptoms in babies in the community. Studies of symptoms in babies dying suddenly and unexpectedly have used case-control comparisons, but the numbers of controls were relatively small, the symptoms were not well defined, and their severity and duration were not recorded. ${ }^{41}$ In studies of babies' symptoms in general practice, information was collected only about babies who were unwell. ${ }^{10} 1213$ In one study the questions were retrospective and there were variations in what parents and doctors meant by a particular symptom. ${ }^{12}$

The present study is part of a larger project aiming to design a system to help parents and doctors assess the severity of acute illness in babies. Data have also been collected from 709 babies presenting to hospital. Any system that is going to help parents and professionals to decide when a baby's symptoms warrant medical attention must have high specificity in order to avoid increasing both parental anxiety and the workload on the primary health care team. It must therefore take account of the incidence of symptoms within the community. This paper presents data about the incidence, severity, and duration of symptoms in 298 randomly selected infants assessed at home.

\section{Subjects and methods}

ENROLMENT OF SUBJECTS

The study aimed to assess 300 infants. Babies were enrolled postnatally at the maternity hospital in Cambridge and were eligible for the study if (i) the baby was born at more than 36 weeks' gestation and was healthy at birth, and (ii) the mother was English speaking and lived within five miles of the hospital. Babies were randomly selected from the birth register. The enrolment schedule ensured an even distribution of assessments across postnatal age ( 1 to 25 weeks) and season.

In 275 cases the mother who was initially approached agreed to participate. When a mother refused a substitute was randomly selected from other babies born on the same day. Thirteen infants could not be assessed (12 failed to keep the appointment and one died suddenly at home). Twelve were replaced. One mother failed to keep her appointment on the last day of the study. Thus 299 infants were assessed.

ASSESSMENT

Infants between 1 and 25 weeks of age were seen 
Table 1 Definitions of symptoms

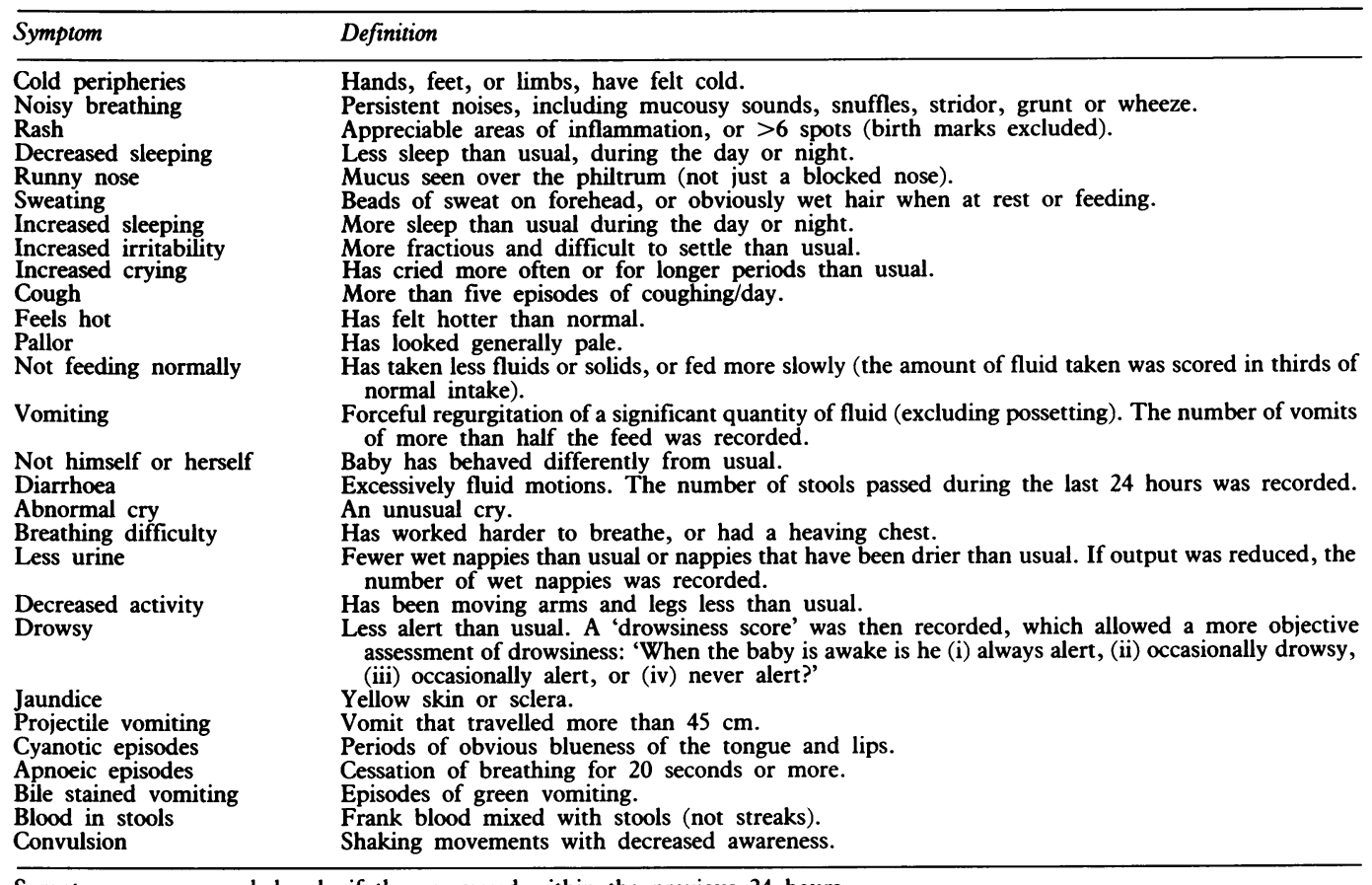

Symptoms were recorded only if they occurred within the previous 24 hours.

at home by one observer (AJT) between 1 April 1986 and 31 March 1987. The mother was asked whether she was concerned about the baby's health, and whether the baby was ill. She was then questioned in detail about 28 symptoms, which were predefined in a study manual (table 1). A symptom was recorded only if it had occurred during the previous 24 hours. The mother was asked about its total duration, how long it had been at its present severity, and her perception of the current severity on a four point scale indicating normal, mild, moderate, and severe (with normal taken as the parent's perception of what is normal for babies generally). Data about the baby's birth and family circumstances were also collected. Details of all hospital admissions, and of medication, immunisations, and consultations with the general practitioner (excluding routine contacts) within specified periods were recorded.

A detailed and standardised examination covering 48 signs was also carried out. At the end of the assessment, the assessor's impression of the degree of illness was recorded on a five point scale indicating well, or mildly, moderately, or severely ill, or requiring resuscitation. The babies were visited on the next three days by a second investigator (JMT) who recorded medical contacts and changes in their conditions.

\section{Results}

Of the 299 infants, one was excluded from analysis as he was in hospital at the time of assessment with pneumonia that necessitated mechanical ventilation.

THE STUDY POPULATION

Similar numbers of infants were seen at each month of age and in each season. One baby was diagnosed as having congenital heart disease after enrolment. Just over half of the babies were girls; $125(42 \%)$ were the only child in the house; in $106(36 \%)$ there was one other child, in $52(17 \%)$ there were two others, and in 16 $(5 \%)$ there were three or more. Of the parents $254(85 \%)$ were married, 27 (9\%) were cohabiting, and $17(6 \%)$ were living alone or with relatives. Fifteen $(5 \%)$ of the mothers were less than 20 years old, $61(21 \%)$ were $20-24$, and 42 $(11 \%)$ were 35 years or more. The social class distribution was social class I-22\%, II-19\%, III (non-manual)-9\%, III (manual)-27\%, IV $-14 \%$, and V-2\%. In $7 \%$ there was insufficient information to code the social class. ${ }^{14}$ In nearly half the families at least one parent smoked.

VISITS TO THE DOCTOR IN THE PREVIOUS WEEK Forty three babies (14\%) had been seen by a doctor during the previous week, in four cases more than once. Four babies $(1 \%)$ were seen on the day of assessment, and two of these had also been seen during the previous week. Four babies (1\%) had appointments later that day. One baby had been discharged from hospital on the morning of the assessment.

DRUGS AND IMMUNISATIONS

Eighteen babies $(6 \%)$ had received antipyretics in the previous 24 hours and nine $(3 \%)$ had received antibiotics in the previous 72 hours. Fifty five (18\%) had been immunised in the previous fortnight, eight $(3 \%)$ within the previous 24 hours.

ASSESSOR'S IMPRESSION OF ILLNESS

Two hundred and thirty nine babies $(80 \%)$ were graded as well, and $51(17 \%)$ as mildly ill. Only 
eight $(3 \%)$ were considered moderately ill. Of these, three were under review by their general practitioner for respiratory infections, and one had whooping cough. Two were seen by their general practitioner later the same day; one developed measles, and the other was admitted to hospital eight hours after the assessment with a urinary tract infection and septicaemia. No babies were considered to be seriously ill at the time of the assessment.

\section{MOTHERS' CONCERNS ABOUT THE BABIES'} HEALTH

Forty seven mothers (16\%) mentioned one or more of 22 different concerns (table 2). Thirty three mentioned one concern, 11 mentioned two, two mentioned three, and one mentioned four. Twenty three mothers (8\%) thought their baby was ill at the time of the assessment or had been ill within the last 24 hours.

Table 2 Problems that concerned the mother on initial questioning

\begin{tabular}{ll}
\hline Problem & $\begin{array}{l}\text { No(\%) of babies } \\
(n=298)\end{array}$ \\
\hline Noisy breathing & $10(3)$ \\
Common cold & $10(3)$ \\
Increased irritability & $5(2)$ \\
Not feeding normally & $4(1)$ \\
Cough & $4(1)$ \\
Local infection & $4(1)$ \\
Breathing difficulty & $3(1)$ \\
Constipation & $3(1)$ \\
Vomiting & $3(1)$ \\
Rash & $3(1)$ \\
Runny nose & $2(1)$ \\
Conjunctivitis & $2(1)$ \\
Colic & $2(1)$ \\
Apnoeic episode & $1(<1)$ \\
Diarrhoea & $1(<1)$ \\
Decreased sleeping & $1(<1)$ \\
Feels hot & $1(<1)$ \\
Chest infection & $1(<1)$ \\
Not himself/herself & $1(<1)$ \\
Weight gain & $1(<1)$ \\
Others & $3(1)$ \\
\hline
\end{tabular}

\section{INCIDENCE OF SYMPTOMS ON DIRECT} QUESTIONING

The mothers of 241 babies ( $81 \%$ ) reported one to 15 symptoms present in the previous 24 hours. Sixty nine (23\%) reported one symptom, $49(16 \%)$ reported two symptoms, $40(13 \%)$ reported three symptoms, and $83(28 \%)$ reported four or more symptoms. Only 57 babies (19\%) were wholly free of symptoms. The number of mothers reporting symptoms when directly questioned was five times the number who expressed 'concerns' about their babies' health, and 10 times the number who thought their babies were ill.

Table 3 shows the incidence, duration, and severity of symptoms. The commonest symptoms were cold peripheries (39\%), noisy breathing $(30 \%)$, rash $(23 \%)$ decreased sleeping $(20 \%)$, runny nose $(18 \%)$, and sweating $(17 \%)$. Of 88 mothers reporting noisy breathing, 82 $(93 \%)$ were referring to snuffles or upper airway 'mucousy' noises; three (3\%) mentioned 'chesty' sounds, two (2\%) wheezy noises, and one stridor. Despite the high incidence of noisy breathing, coughing was reported in only $10 \%$ of babies and breathing difficulties in $3 \%$. Feeding disturbances were reported in 24 babies $(8 \%)$. Details of the babies' intakes were obtained in 288 cases (97\%). Sixteen babies $(6 \%)$ had taken less fluid than normal (including five whose mothers had initially reported no feeding disturbance). Eleven had taken more than two thirds of their normal fluids and two had taken between one and two thirds. In three the actual amount taken was unknown. None had taken less than a third. In 11 cases $(4 \%)$ the report of feeding disturbance referred to a change in the feeding pattern or to a reduction in the amount of solids taken, and not to a reduction in the fluid intake.

Symptoms that might be considered serious (such as decreased activity, drowsiness, apnoeic attacks, and projectile vomiting) were reported

Table 3 Incidence, severity, and duration of 28 symptoms ascertained by direct questioning of mothers of 298 babies

\begin{tabular}{|c|c|c|c|c|}
\hline \multirow[t]{2}{*}{ Symptom } & \multirow[t]{2}{*}{ Total No (\%) } & \multirow{2}{*}{$\begin{array}{l}\text { No (\%) with moderate } \\
\text { or severe symptoms }\end{array}$} & \multicolumn{2}{|c|}{ Duration of symptoms } \\
\hline & & & $\begin{array}{l}\text { No }(\%) \\
\leqslant 3 \text { days }\end{array}$ & $\begin{array}{l}\text { No (\%) } \\
\text { 'always present' }\end{array}$ \\
\hline $\begin{array}{l}\text { Cold peripheries } \\
\text { Noisy breathing } \\
\text { Rash } \\
\text { Decreased sleeping } \\
\text { Runny nose } \\
\text { Sweating } \\
\text { Increased sleeping } \\
\text { Increased irritability } \\
\text { Increased crying } \\
\text { Cough } \\
\text { Feels hot } \\
\text { Pallor } \\
\text { Not feeding normally } \\
\text { Vomiting } \\
\text { Not himself or herself } \\
\text { Diarrhoea } \\
\text { Abnormal cry } \\
\text { Breathing difficulty } \\
\text { Less urine } \\
\text { Decreased activity } \\
\text { Drowsy } \\
\text { Jaundice } \\
\text { Projectile vomiting } \\
\text { Cyanotic episodes } \\
\text { Apnoeic episodes } \\
\text { Bile stained vomiting } \\
\text { Blood in stools } \\
\text { Convulsions }\end{array}$ & $\begin{array}{l}117(39) \\
88(30) \\
68(23) \\
61(20) \\
53(18) \\
51(17) \\
45(15) \\
45(15) \\
43(14) \\
42(14) \\
36(12) \\
30(10) \\
24(8) \\
24(8) \\
21(7) \\
16(5) \\
13(4) \\
10(3) \\
9(3) \\
7(2) \\
5(2) \\
4(1) \\
3(1) \\
2(1) \\
2(1) \\
0 \\
0 \\
0\end{array}$ & $\begin{aligned} & 3(1) \\
& 17(6) \\
& 16(5) \\
& 11(4) \\
& 11(4) \\
& 6(2) \\
& 1(<1) \\
& 13(4) \\
& 18(6) \\
& 4(1) \\
& 22(7) \\
& 5(2) \\
& 5(2) \\
& 7(2) \\
& 8(3) \\
& 4(1) \\
& 4(1) \\
& 7(2) \\
& 1(<1) \\
& 2(1) \\
& 1(<1) \\
& 0 \\
& 1(<1) \\
& 0 \\
& 1(<1) \\
& 0 \\
& 0 \\
& 0\end{aligned}$ & $\begin{aligned} & 7(2) \\
& 20(7) \\
& 19(6) \\
& 23(8) \\
& 22(7) \\
& 7(2) \\
& 18(6) \\
& 18(6) \\
& 18(6) \\
& 16(5) \\
& 20(7) \\
& 4(1) \\
& 17(6) \\
& 10(3) \\
& 8(3) \\
& 7(2) \\
& 5(2) \\
& 3(1) \\
& 4(1) \\
& 4(1) \\
& 5(2) \\
& 0 \\
& 2(1) \\
& 0 \\
& 0 \\
& 0 \\
& 0 \\
& 0\end{aligned}$ & $\begin{aligned} & 105(35) \\
& 34(11) \\
& 18(6) \\
& 7(2) \\
& 1(<1) \\
& 36(12) \\
& 1(<1) \\
& 2(1) \\
& 1(<1) \\
& 4(1) \\
& 9(3) \\
& 23(8) \\
& 0 \\
& 7(2) \\
& 1(<1) \\
& 2(1) \\
& 3(1) \\
& 5(2) \\
& 0 \\
& 0 \\
& 0 \\
& 3(1) \\
& 1(<1) \\
& 2(1) \\
& 1(<1) \\
& 0 \\
& 0 \\
& 0\end{aligned}$ \\
\hline
\end{tabular}


in $1-2 \%$ of babies. Bile stained vomiting, blood in the stools, and convulsions did not occur. Neonatal jaundice was reported in four babies.

\section{DURATION OF SYMPTOMS}

The duration of symptoms (table 3) was divided into 'recent onset' (present for three days or less), 'intermediate' (present for more than four days but less than 14 days) and 'always present' (present since birth or for more than 14 days).

\section{SYMPTOMS OF RECENT ONSET}

Although $81 \%$ of the babies had symptoms, only $38 \%$ had symptoms of recent onset (table 3). The commonest symptom, cold peripheries, was reported in $39 \%$ of babies, but was of recent onset in only $2 \%$. Sweating was reported in $17 \%$ but was recent in only $2 \%$. Pallor was reported in $10 \%$, but was recent in only $1 \%$. The symptoms with a high incidence of acute presentation were: drowsiness in $5 / 5(100 \%)$, not feeding normally in $17 / 24(71 \%)$, projectile vomiting in $2 / 3(66 \%)$, decreased activity in $4 / 7(57 \%)$, and feeling hot in $20 / 36(56 \%)$.

\section{SYMPTOMS REPORTED AS 'ALWAYS PRESENT'}

A third of the symptoms were reported as always present (table 3). The commonest were cold peripheries in $35 \%$ of babies, sweating in $12 \%$, noisy breathing in $11 \%$, pallor in $8 \%$, and rash in $6 \%$. Pallor and sweating were reported as always present because mothers thought babies were pale when asleep or tired and because the babies sweated at the back of the head when feeding. Potentially serious symptoms such as cyanosis, apnoea, and breathing difficulties were occasionally reported as always present; one baby had cyanotic heart disease, two mothers gave doubtful histories, and four mothers said their babies choked with feeds and had difficulty breathing during or after such episodes. Symptoms such as passing less urine, decreased activity, not feeding normally, increased irritability, increased crying, and increased sleeping could not (by definition) be reported as present since birth. They were rarely reported as being present for longer than two weeks.

\section{SEVERITY OF SYMPTOMS}

Only $21 \%$ of the symptoms reported were graded by the mother as moderate or severe (table 3). The symptoms most likely to be graded as moderate or severe were: breathing difficulty in $7 / 10(70 \%)$, feels hot in $22 / 36$ $(61 \%)$, apnoeic episodes in $1 / 2(50 \%)$, and increased crying in 18/43 (43\%). Those least likely to be graded as moderate or severe were: cold peripheries in $3 / 17(1 \%)$, increased sleeping in $1 / 45(2 \%)$, less urine in $1 / 9(11 \%)$, coughing in $4 / 42(10 \%)$ and sweating in $6 / 51(11 \%)$.

\section{Discussion}

This paper presents accurately defined data about the symptoms affecting 298 babies under 6 months of age seen at home. The results show that the overall incidence of symptoms in this age group is high, with many babies having several symptoms. Some of the symptoms studied were common, and had been present for many days. The mothers often considered these to be 'normal' and rarely a cause for concern.

The study population is typical of the national population for the most important social factors such as number of children in the family, single parents, and smoking, and enough babies were assessed to give good $95 \%$ confidence intervals for the incidence of symptoms. The cohort is weighted towards social class I ( $22 \%$, compared with $4 \cdot 5 \%$ nationally), but regression analysis taking into account the season, month of assessment, social class, mother's age, number of children in the house, parental smoking, whether the mother worked, baby's age and feeding method (breast or bottle and whether taking solids), did not show any significant effect of social class on the reporting of individual symptoms. Season and the month of assessment were the factors that influenced reporting most often, with an increased incidence of cold peripheries, decreased sleeping, runny nose, increased irritability, increased crying, cough, sweating, and rash in the winter months. The remaining factors in the analysis affected the incidence of one or two symptoms each (for example, parental smoking was related to an increased incidence of vomiting and sweating), although when comparatively small numbers of babies had the symptom (that is, less than 30 babies) it was rarely possible to show any significant effects. Further analyses are planned to explore in more detail the effect of various factors on the reporting of symptoms. If the study were to be repeated in a community where any of the factors affecting incidence of symptoms were more common (for example in a population with more smokers) the incidence of symptoms would vary in accordance with these differences. Nevertheless, the common symptoms would remain common and the rarer symptoms would remain rare. The incidence of symptoms would still be high (perhaps higher) and the comparative importance of individual symptoms as indicators of disease would be similar.

When asked whether they had any worries about their baby's health, $16 \%$ of mothers in the study expressed a concern; $8 \%$ thought that their babies were ill. When questioned specifically about the presence of individual symptoms, a third reported symptoms of recent onset. A fifth of the babies had symptoms that the mothers thought were important (graded as moderate or severe). The assessor considered that $20 \%$ of the babies were mildly or moderately ill at the time of the assessment. Thus it seems that on any day about a fifth of the babies of this age have symptoms that might be related to minor illness. Serious illnesses, however, are rare. The high incidence of symptoms and minor illness is probably a reflection of the large numbers of infective agents encountered by babies in the first few months of life, to which they must mount an immunological response. 
Given that babies commonly have symptoms, the assessment of their comparative importance can be difficult. Various authors have suggested lists of serious or sinister symptoms. ${ }^{4-11}$ Stanton et al highlighted 12 'major' symptoms that warranted medical attention the same day. These were wheezing, noisy or altered breathing, cough, diarrhoea or vomiting, unusual drowsiness, irritability or excessive crying, an altered character to the cry, being off feeds, or having difficulty feeding. The definitions used are not described and thus any comparison of their data and our own should be cautious; most of these 'major' symptoms, however, seem to be too common in babies of this age to be useful individual predictors of serious outcome. Only drowsiness and altered cry were uncommon symptoms in our study, being present in less than $5 \%$ of the babies. Reduced feeding was included in the list given by Stanton et al, but has been criticised as being too common in general practice to be useful. ${ }^{10}$ The results of the present study, however, suggest that a baby taking less than two thirds of the normal fluid intake is rare in the community, and no baby was taking less than one third of the normal intake. This degree of reduction in fluid intake may therefore be a useful symptom.

Some studies have used the non-specific 'presence of symptoms' as an indicator of illness in babies. Vague pointers such as 'symptoms in an infant less than 6 months old' and 'the presence of several symptoms' are, however, obviously impractical as indicators of the need for medical attention ${ }^{10} ; 41 \%$ of the babies in our study had three or more symptoms at the time of the assessment.

The incidence of symptoms in this study is higher than has been reported for control babies in previous studies. ${ }^{41}$ This may be because of a difference in methods. Our enquiry covered more symptoms, and direct and detailed questioning about each symptom using strict definitions is likely to have increased the rate of reporting compared with the indirect and less specific methods used in previous studies.

Some specific symptoms that are known to indicate serious conditions are rare in the community. Drowsiness, decreased activity, projectile vomiting, cyanosis, and apnoea were rare. Bile stained vomiting, frank blood in the stools, and convulsions were not found in this study. These symptoms warrant an urgent medical opinion.

When taking a history, simply asking the mother what she is concerned about will not provide accurate data. The number of mothers who reported concerns about their babies was less than a fifth of the number who reported symptoms when questioned directly. Detailed and specific questions should be asked about each symptom. It is important to differentiate between symptoms that are of recent onset, or are severe, and may be related to acute illness, and those that have been present for some time, are less severe, and may be part of the normal symptom profile in the community. Pallor and sweating were often reported as being always present, and were graded as normal or mild because mothers reported healthy babies look- ing pale when asleep and sweating when feeding. In contrast, pallor or sweating that were severe or of recent onset were rare ( $2 \%$ or less) and thus more likely to be signs of illness. The commonest symptom, cold peripheries, was reported as always present in $90 \%$ of the babies who had the symptom. It was rarely described as severe, and was the only symptom present in $14 \%$ of those in whom it was reported. This suggests that the symptom is normal for infants in this age group.

The degree of disturbance may need to be determined before the importance of a symptom is clear. For example, $8 \%$ of mothers reported feeding disturbances, but only $5 \%$ of babies had taken less fluids than usual and most of these had taken more than two thirds of their normal amount. None had taken less than a third. Grading of symptoms has the added advantage that it encourages careful definition of symptoms. Without specific definitions individual interpretations of symptoms can vary. ${ }^{12}$

There is undoubtedly a need to improve the recognition of illness in babies. The lists of 'serious' symptoms published so far have been unsatisfactory because they have not taken account of the high incidence of symptoms in the community, and the symptoms given have not been clearly defined. ${ }^{4-11}$ Any assessment system used to identify babies with serious illness must have a high sensitivity to ensure that ill babies are not missed, and a high specificity to avoid increasing the workload on the primary health care team. It must take the high background incidence of symptoms into account.

This project has collected carefully defined data about the incidence, duration, and maternal impression of 28 predefined symptoms in a randomly selected group of babies seen at home during one year. The results have shown that symptoms are common in babies in the first 6 months of life, and that they cannot be interpreted without a detailed history. A few individual symptoms are rare in the community and these warrant an urgent medical opinion, but most of the rare symptoms are highly specific, and tend to be associated with particular conditions. Babies developing serious illnesses often have non-specific symptoms that would not be included in a system designed to assess the severity of a baby's illness that was based on these rare specific symptoms alone. A system of assessment based on careful grading of severity of symptoms, or on combinations of symptoms and signs, is likely to be a much more effective tool.

We thank the Baby Illness Research Project Appeal and the Foundation for the Study of Infant Deaths for financial support, and the mothers and babies of Cambridge for their help.

1 McWeeny PM, Emery JL. Unexpected postneonatal deaths (cot deaths) due to recognizable disease. Arch Dis Child 1975;50:191-6.

2 Oakley JR, McWeeny PM, Hayes-Allen M, Emery JL. Possibly avoidable deaths in hospital in the age-group one week to two years. Lancet 1976;

3 Knowelden J, Keeling J, Nicholl JP. A multicentre study of post neonatal mortality. London: DHSS, 1985.

4 Stanton AN, Downham MAPS, Oakley JR, Emery JL, Knowelden J. Terminal symptoms in children dying sudKnowelden J. Terminal symptoms in children dying suddenly and unexpectedly at home: preliminary report of the Med $\mathcal{f}$ 1978;ii:1249-51. 
5 Grabinar D. Terminal symptoms in children dying suddenly and unexpectedly. $\mathrm{Br}$ Med $\mathcal{F}$ 1978;ii:1430. 6 Valentine S. Terminal symptoms in children dying suddenly

7 Struthers J. Terminal symptoms in children dying suddenly and unexpectedly. $\mathrm{Br} \mathrm{Med} \mathcal{F}$ 1978;ii:1430.

8 Greaves D, Boddy A. Terminal symptoms in children dying suddenly and unexpectedly. Br Med $\mathcal{F}$ 1978;ii:1495-6.

9 Valman B. Preventing infant deaths. Br Med F 1985;290: $339-40$.

10 Thurtle OA, Cox P, Fall C, et al. Preventing infant deaths. $\mathrm{Br}$ Med F 1985;290:1434-5.
11 Carpenter RG, Gardener A, Pursall E, McWeeny PM,Emery JL. Identification of some infants at immediate risk of JL. Identification of some infants at immediate risk of
dying unexpectedly and justifying intensive study. Lancet
1979;ii:343-6.

12 Wilson AD, Downham MAPS, Forster DP. Acute illness in infants: a general practice study. $\mathcal{F} R$ Coll Gen Pract 1984;34:155-9.

13 Wright A, Luffingham GH, North D. Prospective study of symptoms and signs in acutely ill infants in general practice. $B r$ Med $\mathcal{F} 1987 ; 294: 1661-2$.

14 Office of Population Censuses and Surveys. Classification of occupations. London: HMSO, 1980. 